\title{
On A 3-Points Inflated Power Series Distributions Characterizations
}

\author{
Rafid S. A. Alshkaki \\ Department of General Requirements, Ahmed Bin Mohammed Military College, Qatar
}

Received October 7, 2019; Revised November 26, 2019; Accepted December 4, 2019

Copyright $@ 2020$ by authors, all rights reserved. Authors agree that this article remains permanently open access under the terms of the Creative Commons Attribution License 4.0 International License

\begin{abstract}
Differential equations are used in modelling many disciplines, in engineering, chemistry, physics, biology, economics, and other fields of sciences, hence can be used to understand and to determine the underlying probabilistic behavior of phenomena through their probability distributions. This paper came to use a simple form of differential equations, namely, the linear form, to determine the probabilistic distributions of some of the most important and popular sub class of discrete distributions used in real-life, the Poisson, the binomial, the negative binomial, and the logarithmic series distributions. A class of finite number of inflated points power series distributions, that contains the Poisson, the binomial, the negative binomial, and the logarithmic series distributions as some of its members, was defined and some of its characteristics properties, along with characterization of the 3-points inflated of these four distributions, through a linear differential equation for their probability generating functions were given. Further, some previous known results were shown to be special cases of our results.
\end{abstract}

Keywords A 3-Points Inflated Power Series Distributions, Probability Generating Function, Linear Differential Equation

\section{Introduction}

A discrete random variable (rv) $\mathrm{Y}$ is said to have a power series distribution (PSD), abbreviate that by writing $\mathrm{Y} \sim \operatorname{PSD}(\theta, \mathrm{g}(\theta))$, if its probability mass function (PMF) is given by;

$$
\mathrm{P}(\mathrm{Y}=\mathrm{y})=\frac{\mathrm{a}_{\mathrm{y}} \theta^{\mathrm{y}}}{\mathrm{g}(\theta)}, \mathrm{y} \in \mathrm{T}
$$

where $g(\theta)=\sum_{y \in T} a_{y} \theta^{y}$ is a power series for $\theta \in \Omega=$ $\{\theta ; 0<\theta<\omega\}, \omega$ is the radius of convergence of $g(\theta)$, and $\mathrm{a}_{\mathrm{y}} \geq 0$ for all $\mathrm{y} \in \mathrm{T} \subseteq \mathrm{I}=\{0,1,2, \ldots\}$. The function $g(\theta)$ is called the series defining function (SDF). Table (1) gives a summary of parameters, PMFs, and SDFs of these distributions. See Abdul-Razak and Patil $(1986,1994)$, and Johnson et al (2005) for further details.

Let $\alpha \in(0,1)$ be an extra proportion added to the proportion of zero of the rv $\mathrm{Y}$, then the rv $\mathrm{X}$ defined by;

$$
P(X=x)=\left\{\begin{array}{c}
\alpha+(1-\alpha) P(Y=0), x=0 \\
(1-\alpha) P(Y=x), x=1,2,3, \ldots \\
0, \quad \text { otherwise }
\end{array}\right.
$$

is said to have a zero-inflated PSD.

\begin{tabular}{|c|c|c|c|c|c|c|}
\hline No & Name & Parameters & PMF & $T$ & SDF & $w$ \\
\hline 1 & Poisson & $\theta$ & $\frac{\theta^{y}}{y !} e^{-\theta}$ & $\{0,1,2, \ldots\}$ & $e^{\theta}$ & $\infty$ \\
\hline 2 & Binomial & $\begin{array}{c}n \text { and } p \\
\left(p=\frac{\theta}{1+\theta}\right)\end{array}$ & $\left(\begin{array}{l}n \\
y\end{array}\right) p^{y}(1-p)^{n-y}$ & $\{0,1,2, \ldots, n\}$ & $(1+\theta)^{n}$ & $\infty$ \\
\hline 3 & Negative Binomial & $k$ and $\theta$ & $\left(\begin{array}{c}\mathrm{k}+\mathrm{y}-1 \\
\mathrm{y}\end{array}\right) \theta^{\mathrm{y}}(1-\theta)^{\mathrm{k}}$ & $\{0,1,2, \ldots\}$ & $(1-\theta)^{-k}$ & 1 \\
\hline 4 & logarithmic series & $\theta$ & $\frac{\theta^{x}}{-x \log (1-\theta)}$ & $\{1,2,3, \ldots\}$ & $-\log (1-\theta)$ & 1 \\
\hline
\end{tabular}

Table 1. A Summary of Parameters, PMFs, and SDFs of some Power Series Distributions 
The zero inflated PSDs has been studied by many researchers recently. Hu et al (2011), considered zero-inflated and hurdle models and illustrated the consequences of applying models with different distribution assumptions on the data, concluding that if a model used does not closely fit the shape of the data distribution, the estimate of the effect of the intervention may be biased, either over- or underestimating the intervention effect. Phang and Loh (2013), reviewed some related literature zero inflated models for over dispersed count data and provide a variety of examples from different disciplines in the applications of zero inflated models, as well as discussed using different model selection methods in model comparison. Zamri and Zamzuri (2017), review related literature to the zero inflated models, provide a recent development and summary on models for count data with extra zeros. Yang et al (2017), considered comparison of different methods of zero-inflated data analysis and an application in health surveys, and evaluated the performance of several models under different conditions of zero-inflation and dispersion. Sellers and Raim (2017), considered a flexible zero-inflated model to address data dispersion, and illustrates its flexibility, extrapolates the corresponding likelihood ratio test for the presence of significant data dispersion, and highlights various statistical properties and model fit through several examples. Lukusa et al (2017), reviewed studies that considered simultaneously the missing data problem and the zero-inflated feature in modeling zero-inflated data, and discuss their methodologies and results and some potential directions of the future research. In particular, Edwin (2014) considered and studied the number of fetal movement data set, given in Table (2) below, that was collected by Leroux and Puterman (1992), in a study of breathing and body movements in fetal lambs designed to examine the possible change in the amount of pattern of fetal activity during the last two thirds of the gestation period, as a zero inflated PSD model.

Table 2. The number of fetal movement data set, Leroux and Puterman (1992)

\begin{tabular}{|c|c|c|c|c|c|c|c|c|}
\hline $\begin{array}{c}\text { Number of } \\
\text { movements }\end{array}$ & 0 & 1 & 2 & 3 & 4 & 5 & 6 & 7 \\
\hline $\begin{array}{c}\text { Number of } \\
\text { intervals }\end{array}$ & 182 & 41 & 12 & 2 & 2 & 0 & 0 & 1 \\
\hline
\end{tabular}

If we look at the above frequencies of the number of intervals, we notice that not only the number of fetal movement with zeros is inflated but also the ones, and even the twos may be also inflated. This suggests an extension to a finite number of inflated points models.

In this paper, after defining a class of finite number of inflated points power series distributions in Section 2 along with some of its characteristics properties, we considered characterization of the 3-points inflated of the four well-known discrete distributions; namely, the Poisson, binomial, negative binomial, and the logarithmic series distributions, through a linear differential equation for their probability generating functions in Sections 3, 4, 4 and 6, respectively.

\section{A Finite k-Points Inflated Power Series Distributions}

Let the rv $\mathrm{Y} \sim \operatorname{PSD}(\theta, \mathrm{g}(\theta))$ as given in (1) with $\mathrm{T}=\{0,1,2, \ldots\}, \mathrm{k}$ is a non-zero positive finite integer number, $\quad 0<\beta_{\mathrm{i}}<1$ for $\mathrm{i}=0,1,2, \ldots, \mathrm{k}-1 ; \quad 0<$ $\sum_{\mathrm{i}=0}^{\mathrm{k}-1} \beta_{\mathrm{i}}<1$, and $\beta=1-\sum_{\mathrm{i}=0}^{\mathrm{k}-1} \beta_{\mathrm{i}}$, then the rv X defined by;

$$
P(X=x)=\left\{\begin{array}{lc}
\beta_{x}+\beta \frac{a_{x} \theta^{x}}{g(\theta)}, x=0,1,2, \ldots, k-1 \\
\beta \frac{a_{x} \theta^{x}}{g(\theta)}, & x=k, k+1, \ldots \\
0 & \text { otherwise }
\end{array}\right.
$$

is said to have a k-points inflated PSD with parameters $\theta, \beta_{0}, \beta_{1}$, and $\beta_{2}$, abbreviated by $\mathrm{X} \sim \operatorname{kIPSD}\left(\theta, \mathrm{g}(\theta) ; \beta_{0}\right.$, $\left.\beta_{1}, \ldots, \beta_{\mathrm{k}-1}\right)$.

We note that if $\mathrm{k}=1$ in (3), then we have a zero inflated model that given in (1) with $\alpha=\beta_{0}$.

The mean of the rv $X$, with $g^{\prime}(\theta)=\frac{\partial}{\partial \theta} g(\theta)$, is given by;

$$
\begin{aligned}
E(X) & =\sum_{i=0}^{k-1} i \beta_{i}+\theta \beta \frac{g^{\prime}(\theta)}{g(\theta)} \\
& =\sum_{i=0}^{k-1} i \beta_{i}+\beta E(Y)
\end{aligned}
$$

And its variance, with $\mathrm{g}^{\prime \prime}(\theta)=\frac{\partial^{2}}{\partial \theta^{2}} \mathrm{~g}(\theta)$, is given by;

$$
\begin{aligned}
& \operatorname{Var}(\mathrm{X})= \theta \beta\left\{\theta \frac{\mathrm{g}^{\prime \prime}(\theta)}{\mathrm{g}(\theta)}+\frac{\mathrm{g}^{\prime}(\theta)}{\mathrm{g}(\theta)}-\theta \beta\left[\frac{\mathrm{g}^{\prime}(\theta)}{\mathrm{g}(\theta)}\right]^{2}\right\} \\
&+\sum_{\mathrm{i}=0}^{\mathrm{k}-1}\left[\mathrm{i}+\left(1-2 \theta \beta \frac{\mathrm{g}^{\prime}(\theta)}{\mathrm{g}(\theta)}\right)\right] \mathrm{i} \beta_{\mathrm{i}} \\
&-\left[\sum_{\mathrm{i}=0}^{\mathrm{k}-1} \mathrm{i} \beta_{\mathrm{i}}\right]^{2} \\
&=\beta \operatorname{Var}(\mathrm{Y})+\beta(1-\beta) \mathrm{E}(\mathrm{Y})+\sum_{\mathrm{i}=0}^{\mathrm{k}-1}[\mathrm{i}+(1-2 \beta E(\mathrm{Y}))] \mathrm{i} \beta_{\mathrm{i}} \\
&-\left[\sum_{\mathrm{i}=0}^{\mathrm{k}-1} \mathrm{i} \beta_{\mathrm{i}}\right]^{2}
\end{aligned}
$$

Similarly, its probability generating function (PGF) is given by;

$$
\begin{aligned}
G_{X}(t) & =\sum_{i=0}^{k-1} t^{i} \beta_{i}+\theta \beta \frac{g^{\prime}(\theta t)}{g(\theta)} \\
& =\sum_{i=0}^{k-1} t^{i} \beta_{i}+\beta G_{Y}(t)
\end{aligned}
$$

Let $\mu_{\mathrm{r}}^{\prime}$ be the rth moment of the rv $\mathrm{X}$ then for $\mathrm{r}=1,2$, $3, \ldots$; it can be shown that it satisfies the following relation;

$$
\begin{aligned}
\mu_{r+1}^{\prime} & =\sum_{\mathrm{i}=0}^{\mathrm{k}-1} \mathrm{i}^{\mathrm{r}+1} \beta_{\mathrm{i}}+\theta \frac{\mathrm{d}}{\mathrm{d} \theta} \mu_{\mathrm{r}}^{\prime} \\
& +\frac{1}{\beta}\left[\mu_{1}^{\prime}-\sum_{\mathrm{i}=0}^{\mathrm{k}-1} \mathrm{i} \beta_{\mathrm{i}}\right]\left[\mu_{\mathrm{r}}^{\prime}-\sum_{\mathrm{i}=0}^{\mathrm{k}-1} \mathrm{ir}_{\mathrm{i}}\right]
\end{aligned}
$$




\section{Characterization of the 3-Points Inflated Poisson Distribution}

Let $\theta \in \Omega=\{\theta ; 0<\theta<\omega\}$, where $\omega$ is the radius of convergence of $\mathrm{e}^{\theta}$, then the discrete rv $\mathrm{X}$ is said to have a 3-points inflated Poisson distribution with parameters $\theta, \beta_{0}, \beta_{1}$, and $\beta_{2}$, abbreviated by $X \sim 3 \operatorname{IPD}\left(\theta ; \beta_{0}, \beta_{1}, \beta_{2}\right)$, if its PMF is given by;

$$
P(X=x)= \begin{cases}\beta_{x}+\beta \frac{\theta^{x}}{x !} e^{-\theta}, & x=0,1,2 \\ \beta \frac{\theta^{x}}{x !} e^{-\theta}, & x=3,4, \ldots \\ 0, & \text { otherwise }\end{cases}
$$

where $0<\beta_{\mathrm{i}}<1$ for $\mathrm{i}=0,1,2 ; 0<\beta_{0}+\beta_{1}+\beta_{2}<1$, and $\beta=1-\beta_{0}-\beta_{1}-\beta_{2}$. Its PGF, $G(t)$, is given by;

$$
G(t)=\beta_{0}+\beta_{1} t+\beta_{2} t^{2}+\beta e^{\theta(t-1)}
$$

We give below the main result of Section 3 .

Theorem 1: Let $\mathrm{X}$ be a discrete rv taking non-negative integer values, then $X \sim 3 \operatorname{IPD}\left(\theta ; \beta_{0}, \beta_{1}, \beta_{2}\right)$, for some non-zero numbers $\theta, \beta_{0}, \beta_{1}$ and $\beta_{2}$, if and only if its PGF, $G(t)$, satisfies for some arbitrary number $b$ and non-zero numbers a, c, d, and e that;

$$
\mathrm{a} \frac{\partial}{\partial \mathrm{t}} \mathrm{G}(\mathrm{t})=\mathrm{b}+\mathrm{ct}+\mathrm{dt}^{2}+\mathrm{eG}(\mathrm{t})
$$

Proof: (i) Suppose that $X \sim 3 \operatorname{IPD}\left(\theta ; \beta_{0}, \beta_{1}, \beta_{2}\right)$, for some non-zero numbers $\theta, \beta_{0}, \beta_{1}$ and $\beta_{2}$, then its PGF, $\mathrm{G}(\mathrm{t})$, is given by (4), from which we get that;

$$
\beta \mathrm{e}^{\theta(\mathrm{t}-1)}=\mathrm{G}(\mathrm{t})-\beta_{0}-\beta_{1} \mathrm{t}-\beta_{2} \mathrm{t}^{2}
$$

Differentiating (4) with respect to t, we have that;

$$
\frac{\partial}{\partial t} G(t)=\beta_{1}+2 \beta_{2} t+\beta \theta e^{\theta(t-1)}
$$

Hence, using (6) into (7), we get that $\mathrm{G}(\mathrm{t})$ satisfies (5) with; $a=1, b=\beta_{1}-\beta_{0} \theta, c=2 \beta_{2}-\beta_{1} \theta, d=-\beta_{2} \theta$ and $\mathrm{e}=\theta$. If $\theta, \beta_{1}$ and $\beta_{2}$ are chosen such that $\theta \neq \frac{2 \beta_{2}}{\beta_{1}}$, then c is a non-zero number, as well as $\mathrm{a}, \mathrm{d}$ and e since $\theta$ and $\beta_{2}$ are non-zero numbers. Therefore, $G(t)$ satisfies (5) for some arbitrary number $b$ and non-zero numbers $a$, c, $\mathrm{d}$ and e.

(ii) Assume first that $b \neq 0$. Without loss of generality, let us assume that $\mathrm{e}=1$, hence (5) can be written, with $\mathrm{d}$ replaced by $\mathrm{f}$ in order not to be confused with derivative symbol d, as;

$$
\mathrm{a} \frac{\partial}{\partial \mathrm{t}} \mathrm{G}(\mathrm{t})=\mathrm{b}+\mathrm{ct}+\mathrm{ft}^{2}+\mathrm{G}(\mathrm{t})
$$

Now, multiply both sides of (8) by $\mathrm{e}^{\frac{-t}{a}}$, then rearrange them with the using of the derivative reserve product rule, it can be rewritten in the following equivalent form;

$$
\frac{\partial}{\partial t}\left[G(t) \cdot e^{\frac{-t}{a}}\right]=\frac{1}{a}[b+(c+f t) t] e^{\frac{-t}{a}}
$$

from which we get that;

$$
G(t) \cdot e^{\frac{-t}{a}}=\int \frac{1}{a}[b+(c+f t) t] e^{\frac{-t}{a}} d t+k_{1}
$$

where $k_{1}$ is an arbitrary constant. Now with proper integration by parts, the integral given in (9) can be evaluated, we get that;

$$
\begin{gathered}
\int \frac{1}{a}[b+(c+f t) t] e^{\frac{-t}{a}} d t=\left[-b-2 a^{2} f-f^{2}\right] e^{\frac{-t}{a}} \\
-[c t+a(c+2 f t)] e^{\frac{-t}{a}}+k_{2}
\end{gathered}
$$

where $k_{2}$ is an arbitrary constant. Since $G(1)=1$, we have from (9) and (10), with $f$ replaced by $d$, that;

$$
\begin{aligned}
G(t)=- & b-2 a^{2} d-d t^{2}-c t-a(c+2 d t) \\
& +\left[1+b+2 a^{2} d+d+c\right. \\
& +a(c+2 d)] e^{-\frac{1}{a}} \cdot e^{\frac{t}{a}}
\end{aligned}
$$

or equivalently, in the form given by (4) with;

$$
\begin{gathered}
\theta=\frac{1}{a} \\
\beta_{0}=-b-a c-2 a^{2} d \\
\beta_{1}=-c-2 a d \\
\beta_{2}=-d
\end{gathered}
$$

and

$$
\beta=1-\beta_{0}-\beta_{1}-\beta_{2}
$$

Let us consider possible values of the parameters; $\theta, \beta_{0}$, $\beta_{1}, \beta_{2}$ and $\beta$, given by (11) to (15), respectively.

If; $a>0$, then $\theta>0$. If; $-1<d<0$, then $0<\beta_{2}<$ 1 . If $\mathrm{c}$ satisfies that $-2 \mathrm{ad}-1<\mathrm{c}<-2 \mathrm{ad}$, then $0<-c-2$ ad $<1$, that is; $0<\beta_{1}<1$. Let;

$$
\delta_{1}=-\mathrm{ac}-2 \mathrm{a}^{2} \mathrm{~d}
$$

then $\delta_{1}-1<\mathrm{b}<\delta_{1}$ is equivalent to $0<-\mathrm{b}-\mathrm{ac}-$ $2 \mathrm{a}^{2} \mathrm{~d}<1$, and hence $0<\beta_{0}<1$. Let;

$$
\delta_{2}=-\mathrm{c}-\mathrm{d}-2 \mathrm{ad},
$$

then $0<\beta_{0}+\beta_{1}+\beta_{2}<1$ is equivalent to $\delta_{1}+\delta_{2}-$ $1<\mathrm{b}<\delta_{1}+\delta_{2}$. Since the intervals $\left(\delta_{1}+\delta_{2}-1\right.$ ,$\left.\delta_{1}+\delta_{2}\right)$ and $\left(\delta_{1}-1, \delta_{1}\right)$ is overlapping and their intersection $\left(\delta_{1}+\delta_{1}-1, \delta_{1}\right)$ is non-empty, it follows that if $\mathrm{b}$ satisfies that $\delta_{1}+\delta_{1}-1<\mathrm{b}<\delta_{1}$, then $\mathrm{c}$ satisfies that $0<\beta_{0}<1$, and $0<\beta_{0}+\beta_{1}+\beta_{2}<1$, and therefore $\mathrm{G}(\mathrm{t})$ is a PGF of a $\operatorname{3IPD}\left(\theta ; \beta_{0}, \beta_{1}, \beta_{2}\right)$.

If $b=0$, then the solution of (5) leads also to the same result as given in the case when $b \neq 0$, except that $b=0$ in the value $\beta_{0}$ in (12). The values of parameters $\theta$ and $\beta_{2}$ are as given as in the case of $b \neq 0$ above, and we need to consider values of $\beta_{0}$ and $\beta_{1}$ only. We note first, using (12) and (13), that $\beta_{0}=a \beta_{1}$, then we consider the following two cases:

If $a>1$, and since $\beta_{0}=a \beta_{1}$, then $\beta_{1} \leq \beta_{0}$, hence it follows that if $0<\beta_{0}<1$, then $0<\beta_{1}<1$. Now, using (12), (13) and that $\beta_{0}=a \beta_{1}$; then $0<\beta_{0}+\beta_{1}+$ $\beta_{2}<1$ is equivalent to $\frac{a}{1+a} d<\beta_{0}<\frac{a}{1+a}(1+d)$, or equivalently to; 


$$
-2 \mathrm{ad}-\frac{1+\mathrm{d}}{1+\mathrm{a}}<\mathrm{c}<-2 \mathrm{ad}-\frac{\mathrm{d}}{1+\mathrm{a}}
$$

Also, using (12) then $0<\beta_{0}<1$ is equivalent to;

$$
-2 \mathrm{ad}-\frac{1}{\mathrm{a}}<\mathrm{c}<-2 \mathrm{ad}
$$

Since the intervals $\left(-2 \mathrm{ad}-\frac{1+\mathrm{d}}{1+\mathrm{a}},-2 \mathrm{ad}-\frac{\mathrm{d}}{1+\mathrm{a}}\right)$ and $\left(-2 \mathrm{ad}-\frac{1}{\mathrm{a}},-2 \mathrm{ad}\right)$ are overlapping and their non-empty intersection is $\left(-2 \mathrm{ad}-\frac{1+\mathrm{d}}{1+\mathrm{a}},-2 \mathrm{ad}\right)$, it follows that if $\mathrm{c}$ satisfies that $-2 \mathrm{ad}-\frac{1+\mathrm{d}}{1+\mathrm{a}}<\mathrm{c}<-2 \mathrm{ad}$, it satisfies both (16) and (17), and therefore $0<\beta_{0}+\beta_{1}+\beta_{2}<1$, and also $0<\beta_{0}<1$, and hence $0<\beta_{1}<1$.

Finally, if $0<\mathrm{a} \leq 1$, then since $\beta_{0}=\mathrm{a} \beta_{1}$, it follows that $\beta_{0} \leq \beta_{1}$, hence that if $0<\beta_{1}<1$, then $0<\beta_{0}<1$. Now, using (12), (13) and that $\beta_{0}=\mathrm{a} \beta_{1}$; then $0<\beta_{0}+$ $\beta_{1}+\beta_{2}<1$ is equivalent to $\frac{\mathrm{d}}{1+\mathrm{a}}<\beta_{1}<\frac{1+\mathrm{d}}{1+\mathrm{a}}$, or equivalently to;

$$
-2 \mathrm{ad}-\frac{1+\mathrm{d}}{1+\mathrm{a}}<\mathrm{c}<-2 \mathrm{ad}-\frac{\mathrm{d}}{1+\mathrm{a}}
$$

Also, using (13) then $0<\beta_{1}<1$ is equivalent to; $-2 \mathrm{ad}-1<\mathrm{c}<-2 \mathrm{ad}$. Since the interval ( $-2 \mathrm{ad}-$ $\left.\frac{1+\mathrm{d}}{1+\mathrm{a}},-2 \mathrm{ad}-\frac{\mathrm{d}}{1+\mathrm{a}}\right)$ is a subset of $(-2 \mathrm{ad}-1,-2 \mathrm{ad})$, it follows that if $c$ satisfies (18) then it satisfies $0<\beta_{0}+$ $\beta_{1}+\beta_{2}<1$, and also $0<\beta_{1}<1$, and hence $0<\beta_{0}<$ 1 . This completes the proof of the Theorem.

Theorem 1 leads to the following conclusions.

Theorem 2 (Alshkaki (2016a), p 125): Let $X$ be a discrete rv taking non-negative integer values, then $X \sim 2 \operatorname{IPD}\left(\theta ; \beta_{0}, \beta_{1}\right)$, for some non-zero numbers $\theta, \beta_{0}$ and $\beta_{1}$, if and only if its PGF, G(t), satisfying for some arbitrary number $\mathrm{b}$ and non-zero numbers $\mathrm{a}, \mathrm{c}$, and e that;

$$
\mathrm{a} \frac{\partial}{\partial \mathrm{t}} \mathrm{G}(\mathrm{t})=\mathrm{b}+\mathrm{ct}+\mathrm{eG}(\mathrm{t})
$$

Proof: let $\mathrm{d} \rightarrow 0$ in Theorem 1 .

Theorem 3 (Nanjundan and Pasha (2015a), p 141): Let X be a discrete rv taking non-negative integer values, then $X \sim 1 \operatorname{IPD}\left(\theta ; \beta_{0}\right)$, for some non-zero numbers $\theta$ and $\beta_{0}$, if and only if its PGF, G(t), satisfying for some number $b$ staisfying that $0<\mathrm{b}<1$ and non-zero numbers a, and $\mathrm{e}$ that;

$$
\mathrm{a} \frac{\partial}{\partial \mathrm{t}} \mathrm{G}(\mathrm{t})=\mathrm{b}+\mathrm{eG}(\mathrm{t})
$$

Proof: let $\mathrm{c} \rightarrow 0$ and $\mathrm{d} \rightarrow 0$ in Theorem 1 .

\section{Characterization of the 3-Points Inflated Binomial Distribution}

Let $n \in\{3,4,5 \ldots\}$ and $0<p<1$, then the discrete random variable rv $\mathrm{X}$ is said to have a 3-points inflated binomial distribution with parameters $n, p, \beta_{0}, \beta_{1}$, and $\beta_{2}$, abbreviated by $X \sim \operatorname{SIBD}\left(n, p ; \beta_{0}, \beta_{1}, \beta_{2}\right)$, if its PMF is given by;

$$
P(X=x)=\left\{\begin{array}{c}
\beta_{x}+\beta\left(\begin{array}{l}
n \\
x
\end{array}\right) p^{x}(1-p)^{n-x}, x=0,1,2 \\
\beta\left(\begin{array}{l}
n \\
X
\end{array}\right) p^{x}(1-p)^{n-x}, x=3,4, \ldots, n \\
0, \quad \text { otherwise, }
\end{array}\right.
$$

where, $0<\beta_{\mathrm{i}}<1$ for $\mathrm{i}=0,1,2 ; 0<\beta_{0}+\beta_{1}+\beta_{2}<1$, and $\beta=1-\beta_{0}-\beta_{1}-\beta_{2}$. Its PGF is given by;

$$
\mathrm{G}_{\mathrm{X}}(\mathrm{t})=\beta_{0}+\beta_{1} \mathrm{t}+\beta_{2} \mathrm{t}^{2}+\beta(1-\mathrm{p}+\mathrm{pt})^{\mathrm{n}}
$$

On the same lines of the proof of Theorem 1, we give below the main result of Section 4 .

Theorem 4: Let the discrete rv $X$ taking non-negative integer values, then $X \sim \operatorname{3IBD}\left(n, p ; \beta_{0}, \beta_{1}, \beta_{2}\right)$, for some non-zero numbers $n, p, \beta_{0}, \beta_{1}$, and $\beta_{2}$, if and only if, its PGF, G(t), satisfies for some arbitrary number $c$, a positive number $b$, and non-zero numbers $a, d$, e and $f$, that;

$$
(\mathrm{a}+\mathrm{bt}) \frac{\partial}{\partial \mathrm{t}} \mathrm{G}(\mathrm{t})=\mathrm{c}+\mathrm{dt}+\mathrm{et}^{2}+\mathrm{fG}(\mathrm{t})
$$

Proof: (i) Suppose that $X \sim 3 \operatorname{IBD}\left(n, p ; \beta_{0}, \beta_{1}, \beta_{2}\right)$, for some non-zero numbers $n, p, \beta_{0}, \beta_{1}$, and $\beta_{2}$, then its PGF, $G(t)$, is given by (19), from which we get that;

$$
\beta(1-p+p t)^{n}=G(t)-\beta_{0}-\beta_{1} t-\beta_{2} t^{2}
$$

Differentiating (19) with respect to t, we have that;

$$
\frac{\partial}{\partial t} \mathrm{G}(\mathrm{t})=\beta_{1}+2 \beta_{2} \mathrm{t}+\mathrm{np} \beta(1-\mathrm{p}+\mathrm{pt})^{\mathrm{n}-1}
$$

Hence, using (21) into (22), we get that $G(t)$ satisfies (20) with; $\mathrm{a}=1-\mathrm{p}, \mathrm{b}=\mathrm{p}, \mathrm{c}=\left(\beta_{1}+2 \beta_{2}\right)(1-\mathrm{p})-$ $n p \beta_{0}, d=p\left(\beta_{1}(1-n)+2 \beta_{2}\right), \quad e=n p \beta_{2}$ and $f=n p$. Since $0<p<1$, then $b=p$ is a positive number, and that if $p, \beta_{1}$ and $\beta_{2}$ are chosen such that $n \neq \frac{2 \beta_{2}}{\beta_{1}}+1$, then $\beta_{1}(1-n)+2 \beta_{2} \neq 0$, hence $d$ is a non-zero number, as well as a, e and $f$ since $p, n$ and $\beta_{2}$ are non-zero numbers, therefore, $G(t)$ satisfies (20) for some arbitrary number c, a positive number $b$, and non-zero numbers $a, d$, e and $f$.

ii) 1) Assume that $c \neq 0$ and $f \in\{3,4,5, \ldots\}$. Without loss of generality, let us assume that $b=1$, and then with $\mathrm{d}$ replaced by $\mathrm{h}$ in order not to be confused with derivative symbol d; (20) becomes as;

$$
(\mathrm{a}+\mathrm{t}) \frac{\partial}{\partial \mathrm{t}} \mathrm{G}(\mathrm{t})=\mathrm{c}+\mathrm{ht}+\mathrm{et} \mathrm{t}^{2}+\mathrm{fG}(\mathrm{t})
$$

Now multiply both sides of (23) by $(a+t)^{-f-1}$, rearrange them, and using of the rule of the derivative of two product function, it can be rewritten in the following equivalent form;

$$
\frac{\partial}{\partial \mathrm{t}}\left[(\mathrm{a}+\mathrm{t})^{-\mathrm{f}} \mathrm{G}(\mathrm{t})\right]=(\mathrm{a}+\mathrm{t})^{-\mathrm{f}-1}[\mathrm{c}+(\mathrm{h}+\mathrm{et}) \mathrm{t}],
$$

from which, we have that;

$(\mathrm{a}+\mathrm{t})^{-\mathrm{f}} \mathrm{G}(\mathrm{t})=\int(\mathrm{a}+\mathrm{t})^{-\mathrm{f}-1}[\mathrm{c}+(\mathrm{h}+\mathrm{et}) \mathrm{t}] \mathrm{dt}+\mathrm{k}_{1}$

where $\mathrm{k}_{1}$ is an arbitrary constant. Hence, by making the substituting $\mathrm{u}=\mathrm{a}+\mathrm{t}$ in the integral given in (24) and evaluated it, we get that; 


$$
\begin{aligned}
& \int(a+t)^{-f-1}[c+(h+e t) t] d t \\
& =-\frac{2 a^{2} e+a[2 e f t+h(f-2)]}{f(f-1)(f-2)}(a \\
& +t)^{-f} \\
& -\frac{[h(f-2)+e(f-1) t] f t}{f(f-1)(f-2)}(a+t)^{-f} \\
& -\frac{c\left(f^{2}-3 f+2\right)}{f(f-1)(f-2)}(a+t)^{-f}+k_{2}
\end{aligned}
$$

where $\mathrm{k}_{2}$ is an arbitrary constant. Hence, with h replaced by d, we have that;

$$
\begin{aligned}
G(t) & =-\frac{2 a^{2} e+a[2 e f t+d(f-2)]}{f(f-1)(f-2)} \\
& -\frac{[d(f-2)+e(f-1) t] f t}{f(f-1)(f-2)} \\
- & \frac{c\left(f^{2}-3 f+2\right)}{f(f-1)(f-2)}+k_{3}(a+t)^{f}
\end{aligned}
$$

where $\mathrm{k}_{3}$ is an arbitrary constant. Since $\mathrm{G}(1)=1$; we get that;

$$
\begin{gathered}
\mathrm{k}_{3}=\left[1-\frac{2 \mathrm{a}^{2} \mathrm{e}+\mathrm{a}[2 \mathrm{eft}+\mathrm{d}(\mathrm{f}-2)]}{\mathrm{f}(\mathrm{f}-1)(\mathrm{f}-2)}\right](\mathrm{a}+1)^{-\mathrm{f}} \\
-\frac{[\mathrm{d}(\mathrm{f}-2)+\mathrm{e}(\mathrm{f}-1) \mathrm{t}] \mathrm{ft}}{\mathrm{f}(\mathrm{f}-1)(\mathrm{f}-2)}(\mathrm{a}+1)^{-\mathrm{f}} \\
-\frac{\mathrm{c}\left(\mathrm{f}^{2}-3 \mathrm{f}+2\right)}{\mathrm{f}(\mathrm{f}-1)(\mathrm{f}-2)}(\mathrm{a}+1)^{-\mathrm{f}}
\end{gathered}
$$

Therefore, $\mathrm{G}(\mathrm{t})$ can be written in the following form;

$$
\begin{aligned}
& G(t)=\left[-\frac{(a d+c f-3 c)}{(f-1)(f-2)}-\frac{\left(2 a^{2} e+2 a d+2 c\right)}{f(f-1)(f-2)}\right] \\
&+ \frac{(2 d-2 a e-d f)}{(f-1)(f-2)} t+\frac{(e-e f)}{(f-1)(f-2)} t^{2} \\
&+\left[1+\frac{(a d+c f-3 c)}{(f-1)(f-2)}\right. \\
&+\frac{\left(2 a^{2} e+2 a d+2 c\right)}{f(f-1)(f-2)} \\
&-\frac{(2 d-2 a e-d f)}{(f-1)(f-2)} \\
&\left.-\frac{(e-e f)}{(f-1)(f-2)}\right](a+1)^{-f}(a+t)^{f}
\end{aligned}
$$

or equivalently in the form given by (19) with;

$$
\begin{gathered}
\mathrm{n}=\mathrm{f} \\
\mathrm{p}=\frac{1}{\mathrm{a}+1} \\
\beta_{0}=-\frac{\mathrm{c}}{\mathrm{f}}-\frac{\left(\mathrm{adf}+2 \mathrm{a}^{2} \mathrm{e}+2 \mathrm{ad}\right)}{\mathrm{f}(\mathrm{f}-1)(\mathrm{f}-2)}
\end{gathered}
$$

$$
\begin{gathered}
\beta_{1}=-\frac{\mathrm{c}}{\mathrm{f}}-\frac{2 \mathrm{ae}}{(\mathrm{f}-1)(\mathrm{f}-2)} \\
\beta_{2}=-\frac{\mathrm{e}}{\mathrm{f}-2}
\end{gathered}
$$

and

$$
\beta=1-\beta_{0}-\beta_{1}-\beta_{2}
$$

Now we need to check possible values of the parameters of $\mathrm{p}, \beta_{0}, \beta_{1}, \beta_{2}$, and $\beta$ given by (25) to (29), respectively. If $\mathrm{a}>0$, then $\mathrm{p}$ satisfies that $0<\mathrm{p}<1$. Since $\mathrm{f}$ $\in\{3,4,5, \ldots\}$, it follows that if we chose e such that $2-\mathrm{f}<\mathrm{e}<0$ then $0<-\frac{\mathrm{e}}{\mathrm{f}-2}<1$, that is $0<\beta_{2}<1$.

Now, if $\mathrm{d}$ is satisfying that $-(\mathrm{f}-1)-\frac{2 \mathrm{ea}}{\mathrm{f}-2}<\mathrm{d}<-\frac{2 \mathrm{ea}}{\mathrm{f}-2}$, then 2 ea $<-\mathrm{d}(\mathrm{f}-2)<(\mathrm{f}-1)(\mathrm{f}-2)+2$ ea, and hence $0<\frac{-\mathrm{d}(\mathrm{f}-2)-2 \mathrm{ea}}{(\mathrm{f}-1)(\mathrm{f}-2)}<1$, that is $0<\beta_{1}<1$. Next, if we chose $\mathrm{c}$ such that;

$$
-\mathrm{f}-\frac{\left(\mathrm{adf}+2 \mathrm{ea} \mathrm{a}^{2}+2 \mathrm{ad}\right)}{(\mathrm{f}-1)(\mathrm{f}-2)}<\mathrm{c}<-\frac{\left(\mathrm{adf}+2 \mathrm{ea}^{2}+2 \mathrm{ad}\right)}{(\mathrm{f}-1)(\mathrm{f}-2)},
$$

then by noting that $\mathrm{f} \in\{3,4,5, \ldots\}$, (30) reduces to $\frac{\left(\mathrm{adf}+2 e \mathrm{e}^{2}+2 \mathrm{ad}\right)}{\mathrm{f}(\mathrm{f}-1)(\mathrm{f}-2)}<-\frac{\mathrm{c}}{\mathrm{f}}<1+\frac{\left(\mathrm{adf}+2 e \mathrm{a}^{2}+2 \mathrm{ad}\right)}{\mathrm{f}(\mathrm{f}-1)(\mathrm{f}-2)}$, that is $0<$ $-\frac{\left(\mathrm{adf}+2 \mathrm{ea}^{2}+2 \mathrm{ad}\right)}{\mathrm{f}(\mathrm{f}-1)(\mathrm{f}-2)}-\frac{\mathrm{c}}{\mathrm{f}}<1$, or equivalently $0<\beta_{0}<1$. Now, from (26), (27) and (28), we can write;

$$
\begin{aligned}
\beta_{0}+\beta_{1} & +\beta_{2}=-\frac{\left(\mathrm{adf}+2 e \mathrm{a}^{2}+2 \mathrm{ad}\right)}{\mathrm{f}(\mathrm{f}-1)(\mathrm{f}-2)} \\
- & \frac{(\mathrm{d}(\mathrm{f}-2)+2 \mathrm{ae}+\mathrm{e}(\mathrm{f}-1))}{(\mathrm{f}-1)(\mathrm{f}-2)}-\frac{\mathrm{c}}{\mathrm{f}}
\end{aligned}
$$

Therefore, if c chosen such that;

$$
0<-\frac{\left(\mathrm{adf}+2 \mathrm{ea}^{2}+2 \mathrm{ad}\right)}{\mathrm{f}(\mathrm{f}-1)(\mathrm{f}-2)}-\frac{(\mathrm{d}(\mathrm{f}-2)+2 \mathrm{ae}+\mathrm{e}(\mathrm{f}-1))}{(\mathrm{f}-1)(\mathrm{f}-2)}-\frac{\mathrm{c}}{\mathrm{f}}<1,
$$

then $0<\beta_{0}+\beta_{1}+\beta_{2}<1$. Let

$$
\delta_{1}=-\frac{\left(\mathrm{adf}+2 \mathrm{a}^{2} \mathrm{e}+2 \mathrm{ad}\right)}{(\mathrm{f}-1)(\mathrm{f}-2)}
$$

and

$$
\delta_{2}=-\frac{(\mathrm{d}(\mathrm{f}-2)+2 \mathrm{ae}+\mathrm{e}(\mathrm{f}-1)) \mathrm{f}}{(\mathrm{f}-1)(\mathrm{f}-2)}
$$

then (31) is equivalent to $\delta_{1}+\delta_{2}-\mathrm{f}<\mathrm{c}<\delta_{1}+\delta_{2}$, and similarly, (30) is equivalent to $\delta_{1}-\mathrm{f}<\mathrm{c}<\delta_{1}$. Since the intervals $\left(\delta_{1}+\delta_{2}-\mathrm{f}, \delta_{1}+\delta_{2}\right)$ and $\left(\delta_{1}-\mathrm{f}, \delta_{1}\right)$ is overlapping and their intersection $\left(\delta_{1}+\delta_{1}-\mathrm{f}, \delta_{1}\right)$ is non-empty, it follows that if $\mathrm{c}$ is satisfying that $\delta_{1}+\delta_{1}-$ $\mathrm{f}<\mathrm{c}<\delta_{1}$, then $\mathrm{c}$ satisfies both (30) and (31), that is; equivalently $0<\beta_{0}<1$, and $0<\beta_{0}+\beta_{1}+\beta_{2}<1$, respectively, and therefore $\mathrm{G}(\mathrm{t})$ is a PGF of a $\operatorname{3IBD}\left(n, p ; \beta_{0}, \beta_{1}, \beta_{2}\right)$. 
2) Assume now that $\mathrm{c} \neq 0, \mathrm{f}>0$ and that $\mathrm{f} \neq 3,4,5, \ldots$, then without loss of generality we can assume that $f=1$, and consider the following cases;

2.a) $b \neq 1$, then on the same line as above, the solution of (20) is given by;

$$
\mathrm{G}(\mathrm{t})=\mathrm{c}_{0}+\mathrm{c}_{1} \mathrm{t}+\mathrm{c}_{2} \mathrm{t}^{2}+\mathrm{c}_{3}(\mathrm{a}+\mathrm{bt})^{\frac{1}{\mathrm{~b}}}
$$

where $c_{0}, c_{1}, c_{2}$ and $c_{3}$ are constants, and hence;

$$
\begin{gathered}
\frac{\partial^{(x)}}{\partial t^{x}} G(t)=c_{3} \frac{1}{b}\left(\frac{1}{b}-1\right)\left(\frac{1}{b}-2\right) \ldots\left(\frac{1}{b}-(x-1)\right) b^{x}(a \\
+b t)^{\frac{1}{b}-x}, x=3,4,5, \ldots
\end{gathered}
$$

from which, since $P(X=x)=\frac{1}{x !} \frac{\partial^{(x)}}{\partial \mathrm{t}^{\mathrm{x}}} \mathrm{G}(0)$, we get that;

$$
P(X=x)=c_{3} \frac{a^{\frac{1}{5}-x} b^{x-1}}{x !} C\left(x, \frac{1}{b}\right), x=3,4,5, \ldots,
$$

where;

$$
C(x, y)=y(y-1)(y-2) \ldots(y-(x-1))
$$

Hence if $0<\mathrm{b}<1$, then $\mathrm{C}\left(\mathrm{x}, \frac{1}{\mathrm{~b}}\right)$ is negative for some $\mathrm{x}=\left[\frac{1}{\mathrm{~b}}\right]+2,\left[\frac{1}{\mathrm{~b}}\right]+3, \ldots$, where $[\mathrm{w}]$ is the largest integral part of $\mathrm{w}$, and is positive for some other values of $\mathrm{x}$, and therefore $\mathrm{P}(\mathrm{X}=\mathrm{x})$ given by (33) is not a PMF regardless of the value of $c_{3}$.

If $b>1$, then $C\left(x, \frac{1}{b}\right)$ is negative for even values of $x$ and positive for odd values, and therefore $\mathrm{P}(\mathrm{X}=\mathrm{x})$ given by (33) is also not a PMF regardless of the value of $c_{3}$, implying that $\mathrm{G}(\mathrm{t})$ is not a PGF in this case.

2.b) $b=1$, then on the same line as above, the solution of (20) is given by;

$$
\mathrm{G}(\mathrm{t})=\mathrm{d}_{0}+\mathrm{d}_{1} \mathrm{t}+\mathrm{d}_{2} \mathrm{t}^{2}+\mathrm{d}_{3} \log (\mathrm{a}+\mathrm{t})+\mathrm{ad}_{3} \operatorname{tlog}(\mathrm{a}+\mathrm{t})
$$

where $d_{0}, d_{1}, d_{2}$ and $d_{3}$ are constants, and hence on the same lines as in case (2.a) we arrive to $P(X=4)=$ $-\frac{d_{3}}{12}\left(\frac{3}{a^{4}}+\frac{5}{a^{2}}\right)$ and $P(X=5)=\frac{d_{3}}{5}\left(\frac{1}{a^{5}}+\frac{1}{a^{5}}\right)$, from which we conclude that one of these values is negative regardless of the value of $d_{3}$, and hence $P(X=x)$ in this case is not a PMF, implying that $G(t)$ given by (35) is not a PGF.

3 ) If $c \neq 0$ and $\mathrm{f}<0$, then the solution of (20), assuming without loss of generality that $b=1$, is given by;

$$
\mathrm{G}(\mathrm{t})=\mathrm{e}_{0}+\mathrm{et}+\mathrm{e}_{2} \mathrm{t}^{2}+\mathrm{e}_{3}(\mathrm{a}+\mathrm{t})^{\mathrm{f}}
$$

where $e_{0}, e_{1}, e_{2}$ and $e_{3}$ are constants, and hence

$$
\frac{\partial^{(x)}}{\partial t^{x}} G(t)=e_{3} C(x, f)(a+t)^{f-x}
$$

where $C(x, f)$ is given by (34). Since $f<0$, it follows that $C(x, f)$ is negative for odd values of $x$ and positive for even values, therefore $\mathrm{P}(\mathrm{X}=\mathrm{x})$ in this case is also not a PMF regardless of the value of $e_{3}$, implying that the function $\mathrm{G}(\mathrm{t})$ given by (36) is not a PGF.

4) Finally, if $c=0$, then we arrive simply, either by solving (20) directly or by letting $\mathrm{c} \rightarrow 0$ in the above proof, which is straightforward, to the same above conclusion.
This completes the proof.

Theorem 4 leads to the following conclusions:

Theorem 5 (Alshkaki (2014b), p 7): Let Z be a discrete rv taking non-negative integer values, then $\mathrm{Z} \sim$ $2 B D\left(n, p ; \beta_{0}, \beta_{1}\right)$, for some non-zero numbers $n, p, \beta_{0}$, and $\beta_{1}$, if and only if its pgf, $G(t)$, satisfying

$$
(\mathrm{a}+\mathrm{bt}) \frac{\partial}{\partial \mathrm{t}} \mathrm{G}(\mathrm{t})=\mathrm{c}+\mathrm{dt}+\mathrm{fG}(\mathrm{t})
$$

for some arbitrary number $c$, a positive number $b$, and non-zero numbers $\mathrm{a}, \mathrm{d}$ and $\mathrm{f}$.

Proof: let $\mathrm{e} \rightarrow 0$ in Theorem 4.

Theorem 6 (Nanjundan and Pasha (2015b), p 1189): Let $\mathrm{Z}$ be a discrete rv taking non-negative integer values, then $\mathrm{Z} \sim 1 \mathrm{BD}\left(\mathrm{n}, \mathrm{p} ; \beta_{0}\right)$, for some non-zero numbers $n, p$ and $\beta_{0}$ if and only if its PGF, G(t), satisfying;

$$
(\mathrm{a}+\mathrm{bt}) \frac{\partial}{\partial \mathrm{t}} \mathrm{G}(\mathrm{t})=\mathrm{c}+\mathrm{fG}(\mathrm{t})
$$

for some arbitrary number $\mathrm{c}$, a positive number $\mathrm{b}$, and non-zero numbers $\mathrm{a}$ and $\mathrm{f}$.

Proof: let $\mathrm{d} \rightarrow 0$ and $\mathrm{e} \rightarrow 0$ in Theorem 4 .

\section{Characterization of the 3-Points Inflated Negative Binomial Distribution}

Let $\mathrm{k}>0$ and $0<\mathrm{p}<1$, then the discrete rv $\mathrm{X}$ is said to have a 3-points inflated negative binomial distribution with parameters $\mathrm{k}, \mathrm{p}, \beta_{0}, \beta_{1}$, and $\beta_{2}$, abbreviated by $X \sim \operatorname{IINBD}\left(\mathrm{k}, \mathrm{p} ; \beta_{0}, \beta_{1}, \beta_{2}\right)$, if its PMF is given by;

$$
\mathrm{P}(\mathrm{X}=\mathrm{x})=\left\{\begin{array}{c}
\beta_{\mathrm{x}}+\beta\left(\begin{array}{c}
\mathrm{k}+\mathrm{x}-1 \\
\mathrm{x}
\end{array}\right) \mathrm{p}^{\mathrm{x}}(1-\mathrm{p})^{\mathrm{k}}, \mathrm{x}=0,1,2 \\
\beta\left(\begin{array}{r}
\mathrm{k}+\mathrm{x}-1 \\
\mathrm{x}
\end{array}\right) \mathrm{p}^{\mathrm{x}}(1-\mathrm{p})^{\mathrm{k}}, \quad \mathrm{x}=3,4, \ldots \\
0, \quad \text { otherwise, }
\end{array}\right.
$$

where $0<\beta_{\mathrm{i}}<1$ for $\mathrm{i}=0,1,2 ; 0<\beta_{0}+\beta_{1}+\beta_{2}<$ 1 , and $\beta=1-\beta_{0}-\beta_{1}-\beta_{2}$. Its PGF is given by;

$$
G_{X}(t)=\beta_{0}+\beta_{1} t+\beta_{2} t^{2}+\beta\left(\frac{1-p}{1-p t}\right)^{k}
$$

Similarly, on the same lines of the proof of Theorem 1 , we give below the main result of Section 5 .

Theorem 7: The discrete rv $\mathrm{X}$ taking non-negative integer values, has a $\operatorname{3INBD}\left(\mathrm{k}, \mathrm{p} ; \beta_{0}, \beta_{1}, \beta_{2}\right)$ for some non-zero numbers $k, p, \beta_{0}, \beta_{1}$, and $\beta_{2}$, if and only if its PGF, G(t), satisfies for some arbitrary number $c$ and non-zero numbers $\mathrm{a}, \mathrm{b}, \mathrm{d}, \mathrm{e}$ and $\mathrm{f}$, such that $\mathrm{bf}<0$;

$$
(\mathrm{a}+\mathrm{bt}) \frac{\partial}{\partial \mathrm{t}} \mathrm{G}(\mathrm{t})=\mathrm{c}+\mathrm{dt}+\mathrm{et}^{2}+\mathrm{fG}(\mathrm{t})
$$

Proof: (i) Suppose that $X \sim \operatorname{3INBD}\left(k, p ; \beta_{0}, \beta_{1}, \beta_{2}\right)$ for some non-zero numbers $k, p, \beta_{0}, \beta_{1}$, and $\beta_{2}$, then its PGF, $\mathrm{G}(\mathrm{t})$, is given by (37), from which we get that;

$$
\beta\left(\frac{1-\mathrm{p}}{1-\mathrm{pt}}\right)^{\mathrm{k}}=\mathrm{G}(\mathrm{t})-\beta_{0}-\beta_{1} \mathrm{t}-\beta_{2} \mathrm{t}^{2}
$$


Differentiating (37) with respect to t, we have that;

$$
\frac{\partial}{\partial t} G(t)=\beta_{1}+2 \beta_{2} t+k p \beta(1-p)^{k}(1-p t)^{-k-1}
$$

Hence, using (39) into (40), we get that $G(t)$ satisfies (38) with; $\mathrm{a}=1, \mathrm{~b}=-\mathrm{p}, \mathrm{c}=\beta_{1}-\mathrm{kp} \beta_{0}, \mathrm{~d}=2 \beta_{2}-$ $\mathrm{p} \beta_{1}(1+\mathrm{k}), \quad \mathrm{e}=-\mathrm{p} \beta_{2}(2+k)$ and $\mathrm{f}=\mathrm{kp}$. Since $0<\mathrm{p}<1$ and $\mathrm{k}>0$ then $\mathrm{bf}=-\mathrm{p}^{2} \mathrm{k}$ is a negative number, and that if $\mathrm{p}, k, \beta_{1}$ and $\beta_{2}$ are chosen such that $\mathrm{p}(1+\mathrm{k}) \neq \frac{2 \beta_{2}}{\beta_{1}}$, then $\mathrm{d}$ is a non-zero number, as well as a, $b$, e and $f$ since $p, k$ and $\beta_{2}$ are non-zero numbers, therefore, $G(t)$ satisfies (38) for some arbitrary number $c$ and non-zero numbers $\mathrm{a}, \mathrm{b}, \mathrm{d}$, e and $\mathrm{f}$, such that $\mathrm{bf}<0$.

ii) Assume first that $c \neq 0$ and that $f>0$, then $b<0$, and therefore, without loss of generality, we can assume that $b=-1$, and then with $d$ replaced by $h$ in order not to be confused with derivative symbol $d$, (38) becomes;

$$
(\mathrm{a}-\mathrm{t}) \frac{\partial}{\partial \mathrm{t}} \mathrm{G}(\mathrm{t})=\mathrm{c}+\mathrm{ht}+\mathrm{et} \mathrm{t}^{2}+\mathrm{fG}(\mathrm{t})
$$

Now, multiply both sides of (41) by $(a-t)^{f-1}$, then rearrange them with the using of the derivative reserve product rule, it can be rewritten in the following equivalent form;

$$
\frac{\partial}{\partial t}\left[(a-t)^{f} G(t)\right]=(a-t)^{f-1}[c+(h+e t) t]
$$

from which we get that;

$$
(\mathrm{a}-\mathrm{t})^{\mathrm{f}} \mathrm{G}(\mathrm{t})=\int(\mathrm{a}-\mathrm{t})^{\mathrm{f}-1}[\mathrm{c}+(\mathrm{h}+\mathrm{et}) \mathrm{t}] \mathrm{dt}+\mathrm{k}_{1}
$$

where $k_{1}$ is an arbitrary constant. Now by making the substituting $\mathrm{x}=\mathrm{a}-\mathrm{t}$ in the integral given in (42) and evaluated it, we get that;

$$
\begin{aligned}
& \int(a-t)^{f-1}[c+(h+e t) t] d t \\
& \quad=-\frac{(a-t)^{f}}{f(f+1)(f+2)}\left\{2 a^{2} e\right. \\
& \quad+a[2 e f t+h(f+2)] \\
& \quad+[h(f+2)+e(f+1) t] f t+c\left(f^{2}+3 f\right. \\
& \quad+2)\}+k_{2}
\end{aligned}
$$

where $\mathrm{k}_{2}$ is an arbitrary constant. Hence, with h replaced by d, we have that;

$$
\begin{gathered}
G(t)=\frac{2 a^{2} e+a[2 e f t+d(f+2)]}{f(f+1)(f+2)} \\
+\frac{[d(f+2)+e(f+1) t] f t}{f(f+1)(f+2)} \\
+\frac{c\left(f^{2}+3 f+2\right)}{f(f+1)(f+2)}+k_{3}(a-t)^{-f}
\end{gathered}
$$

where $\mathrm{k}_{3}$ is an arbitrary constant. Since $\mathrm{G}(1)=1$; we get that;

$$
k_{3}=\left[1-\frac{2 a^{2} e+a[2 e f t+d(f+2)]}{f(f+1)(f+2)}\right](a-1)^{f}
$$

$$
\begin{gathered}
-\frac{[d(f+2)+e(f+1) t] f t}{f(f+1)(f+2)}(a-1)^{f} \\
-\frac{c\left(f^{2}+3 f+2\right)}{f(f+1)(f+2)}(a-1)^{f}
\end{gathered}
$$

Therefore, $\mathrm{G}(\mathrm{t})$ can be written in the form given by (37) with;

$$
\begin{gathered}
k=f \\
p=\frac{1}{a} \\
\beta_{0}=-\frac{c}{f}-\frac{\left(a d f+2 a^{2} e+2 a d\right)}{f(f+1)(f+2)} \\
\beta_{1}=-\frac{d}{f+1}-\frac{2 a e}{(f+1)(f+2)} \\
\beta_{2}=-\frac{e}{f+2}
\end{gathered}
$$

and

$$
\beta=1-\beta_{0}-\beta_{1}-\beta_{2}
$$

Now we need to check possible values of the parameters $k$, $\mathrm{p}, \beta_{0}, \beta_{1}, \beta_{2}$ and $\beta$, given by (43) to (48), respectively.

If $\mathrm{k}=\mathrm{f}$ then $\mathrm{k}>0$. If $\mathrm{a}>1$, then $\mathrm{p}$ satisfies that $0<\mathrm{p}<1$. If; $-\mathrm{f}-2<\mathrm{e}<0$, and recall that $\mathrm{f}>0$, then $0<-\frac{\mathrm{e}}{\mathrm{f}+2}<1$, hence $\beta_{2}$ satisfies that $0<\beta_{2}<1$. If; $-(\mathrm{f}+1)-\frac{2 \mathrm{ae}}{(\mathrm{f}+1)(\mathrm{f}+2)}<\mathrm{d}<-\frac{2 \mathrm{ae}}{(\mathrm{f}+1)(\mathrm{f}+2)}$, then $0<-\frac{\mathrm{d}}{\mathrm{f}+1}-$ $\frac{2 \mathrm{ae}}{(\mathrm{f}+1)(\mathrm{f}+2)}<1$, and hence $\beta_{1}$ satisfies that $0<\beta_{1}<1$. Let;

$$
\delta_{1}=-\frac{\left(\mathrm{adf}+2 \mathrm{a}^{2} \mathrm{e}+2 \mathrm{ad}\right)}{(\mathrm{f}+1)(\mathrm{f}+2)}
$$

then $\delta_{1}-\mathrm{f}<\mathrm{c}<\delta_{1}$ is equivalent to $0<-\frac{\mathrm{c}}{\mathrm{f}}-$ $\frac{\left(\mathrm{adf}+2 \mathrm{a}^{2} \mathrm{e}+2 \mathrm{ad}\right)}{\mathrm{f}(\mathrm{f}+1)(\mathrm{f}+2)}<1$, and hence $\beta_{0}$ given by (45) satisfies that $0<\beta_{0}<1$. Let;

$$
\delta_{2}=-\frac{(2 \mathrm{ae}+2 \mathrm{~d}+\mathrm{df}+\mathrm{e}(\mathrm{f}+1)) \mathrm{f}}{(\mathrm{f}-1)(\mathrm{f}-2)}
$$

then $0<\beta_{0}+\beta_{1}+\beta_{2}<1$ is equivalent to $\delta_{1}+\delta_{2}-$ $\mathrm{f}<\mathrm{c}<\delta_{1}+\delta_{2}$. Since the intervals $\left(\delta_{1}+\delta_{2}-\mathrm{f}, \delta_{1}+\delta_{2}\right)$ and $\left(\delta_{1}-\mathrm{f}, \delta_{1}\right)$ is overlapping and their intersection $\left(\delta_{1}+\delta_{1}-\mathrm{f}, \delta_{1}\right)$ is non-empty, it follows that if c satisfies that $\delta_{1}+\delta_{1}-\mathrm{f}<\mathrm{c}<\delta_{1}$, then c satisfies that $0<\beta_{0}<$ 1 , and $0<\beta_{0}+\beta_{1}+\beta_{2}<1$, and therefore $\mathrm{G}(\mathrm{t})$ is a PFG of a $\operatorname{SINBD}\left(\mathrm{k}, \mathrm{p} ; \beta_{0}, \beta_{1}, \beta_{2}\right)$.

Now, if $f<0$, then $b>0$, and hence we can divided both sides of (38) by (-b) to get;

$$
\left(\mathrm{a}_{1}-\mathrm{t}\right) \frac{\partial}{\partial \mathrm{t}} \mathrm{G}(\mathrm{t})=\mathrm{c}_{1}+\mathrm{d}_{1} \mathrm{t}+\mathrm{e}_{1} \mathrm{t}^{2}+\mathrm{f}_{1} \mathrm{G}(\mathrm{t})
$$

where $a_{1}=-\frac{a}{b}, c_{1}=-\frac{c}{b}, d_{1}=-\frac{d}{b}, e_{1}=-\frac{e}{b} \quad$ and $f_{1}=-\frac{f}{b}$. Since $f_{1}>0$, then (49) has the same form as (41) with a positive coefficient of $G(t)$, and therefore gives the same conclusion as above; that is, $\mathrm{G}(\mathrm{t})$ is the PGF of a $\operatorname{3INBD}\left(k, p ; \beta_{0}, \beta_{1}, \beta_{2}\right)$. 
Next, if $c=0$, then the solution of (38) leads also to the same result as in the case when $\mathrm{c} \neq 0$, with $\mathrm{c}=0$ in the value $\beta_{0}$ in (45). The values of parameters $k, p$, and $\beta_{2}$ are as given as in the case of $c \neq 0$ above, and we need to consider values of $\beta_{0}$ and $\beta_{1}$ only. We note first that, using (45) and (46) we have that $\beta_{0}=\frac{a}{f} \beta_{1}$, then we consider the following two cases:

If $\mathrm{a} \leq \mathrm{f}$, then since $\beta_{0}=\frac{\mathrm{a}}{\mathrm{f}} \beta_{1}$, then $\beta_{0} \leq \beta_{1}$, hence it follows that if $0<\beta_{1}<1$ then $0<\beta_{0}<1$. Now, using (47) and that $\beta_{0}=\frac{\mathrm{a}}{\mathrm{f}} \beta_{1}$; then $0<\beta_{0}+\beta_{1}+\beta_{2}<1$, is equivalent to $\frac{\mathrm{e}}{\mathrm{f}+2} \frac{\mathrm{f}}{\mathrm{f}+\mathrm{a}}<\beta_{1}<\left(1+\frac{\mathrm{e}}{\mathrm{f}+2}\right) \frac{\mathrm{f}}{\mathrm{f}+\mathrm{a}}$. Since $-\mathrm{f}-2<$ $\mathrm{e}<0$, it follows that $\left(0,\left(1+\frac{\mathrm{e}}{\mathrm{f}+2}\right) \frac{\mathrm{f}}{\mathrm{f}+\mathrm{a}}\right)$ is a subset of $\left(\frac{\mathrm{e}}{\mathrm{f}+2} \frac{\mathrm{f}}{\mathrm{f}+\mathrm{a}},\left(1+\frac{\mathrm{e}}{\mathrm{f}+2}\right) \frac{\mathrm{f}}{\mathrm{f}+\mathrm{a}}\right)$, hence if $0<\beta_{1}<\left(1+\frac{\mathrm{e}}{\mathrm{f}+2}\right) \frac{\mathrm{f}}{\mathrm{f}+\mathrm{a}}$ then $0<\beta_{0}+\beta_{1}+\beta_{2}<1$. Now since $0<\beta_{1}<(1+$ $\left.\frac{\mathrm{e}}{\mathrm{f}+2}\right) \frac{\mathrm{f}}{\mathrm{f}+\mathrm{a}}$ is equivalent to;

$$
-\frac{2 a e}{f+2}-\left(1+\frac{e}{f+2}\right) \frac{f(f+1)}{f+a}<d<-\frac{2 a e}{f+2}
$$

It follows that, if $d$ is satisfies (50), then $0<\beta_{1}<1$, hence also $0<\beta_{0}<1$ and $0<\beta_{0}+\beta_{1}+\beta_{2}<1$.

Finally, if a $>\mathrm{f}$, then $\beta_{1}<\beta_{0}$, and hence if we deal with $\beta_{0}$ on the same lines as we did with $\beta_{1}$ in the case of $\mathrm{a} \leq \mathrm{f}$, we arrive to exactly the same conclusion, namely, if $\mathrm{d}$ is satisfies (50) also, then $0<\beta_{0}<1$, and hence $0<\beta_{1}<1$ and $0<\beta_{0}+\beta_{1}+\beta_{2}<1$. This completes the proof of the Theorem.

Theorem 7 leads to the followings.

Theorem 8 (Alshkaki (2016c), p 3): Let X be a discrete rv taking non-negative integer values, then $\mathrm{X} \sim$ $3 \operatorname{INBD}\left(k, p ; \beta_{0}, \beta_{1}\right)$, for some non-zero numbers $\mathrm{k}, \mathrm{p}, \beta_{0}$ and $\beta_{1}$ if and only if its PGF satisfies for some arbitrary number $c$ and non-zero numbers $a, b, d$ and $f$, such that $\mathrm{bf}<0$, that;

$$
(\mathrm{a}+\mathrm{bt}) \frac{\partial}{\partial \mathrm{t}} \mathrm{G}(\mathrm{t})=\mathrm{c}+\mathrm{dt}+\mathrm{fG}(\mathrm{t})
$$

Proof: Just let $\mathrm{e} \rightarrow 0$ in Theorem 7.

Theorem 9 (Suresh et al (2015), p 512): Let X be a discrete rv taking non-negative integer values, then $\mathrm{X} \sim \operatorname{ZINBD}\left(\mathrm{k}, \mathrm{p} ; \beta_{0}\right)$, for some non-zero numbers $k, p$ and $\beta_{0}$ if and only if its PGF G(t) satisfying

$$
(\mathrm{a}+\mathrm{bt}) \frac{\partial}{\partial \mathrm{t}} \mathrm{G}(\mathrm{t})=\mathrm{c}+\mathrm{fG}(\mathrm{t})
$$

for some arbitrary number $\mathrm{c}$ and non-zero numbers $\mathrm{a}, \mathrm{b}$ and $\mathrm{f}$, such that $\mathrm{bf}<0$;

Proof: Just $d \rightarrow 0$ and $\mathrm{e} \rightarrow 0$ in Theorem 7 .

\section{Characterization of the 3-Points Inflated Logarithmic Series Distribution}

Let $0<\theta<1$, then the discrete $\mathrm{rv} X$ is said to have a 3-points inflated LSD with parameters $\theta, \beta_{0}, \beta_{1}$, and $\beta_{2}$, abbreviated by $\mathrm{X} \sim \operatorname{3ILSD}\left(\theta ; \beta_{0}, \beta_{1}, \beta_{2}\right)$, if its $\operatorname{PMF}$ is given by;

$$
P(X=x)= \begin{cases}\beta_{0}, & x=0, \\ \beta_{x}+\beta \frac{\theta^{x}}{-x \log (1-\theta)}, \quad x=1,2, \\ \beta \frac{\theta^{x}}{-x \log (1-\theta)}, & x=3,4, \ldots, \\ 0, & \text { otherwise, }\end{cases}
$$

where $0<\beta_{\mathrm{i}}<1$ for $\mathrm{i}=0,1,2 ; 0<\beta_{0}+\beta_{1}+\beta_{2}<$ 1 , and $\beta=1-\beta_{0}-\beta_{1}-\beta_{2}$. Its PGF is given by;

$$
G_{X}(t)=\beta_{0}+\beta_{1} t+\beta_{2} t^{2}+\beta \frac{\log (1-\theta t)}{\log (1-\theta)}
$$

Finally, on the same lines of the proof of Theorem 1, we give below the main result of Section 6 .

Theorem 10: The discrete rv $\mathrm{X}$ taking non-negative integer values, has a $3 \operatorname{ILSD}\left(\theta ; \beta_{0}, \beta_{1}, \beta_{2}\right)$ for some non-zero numbers $\theta, \beta_{0}, \beta_{1}$, and $\beta_{2}$, if and only if its PGF, $G(t)$, satisfies for some non-zero numbers $a, b, c, d$ and e that;

$$
(\mathrm{a}+\mathrm{bt}) \frac{\partial}{\partial \mathrm{t}} \mathrm{G}(\mathrm{t})=\mathrm{c}+\mathrm{dt}+\mathrm{et} \mathrm{t}^{2}
$$

Proof: (i) Suppose that $X \sim 3 \operatorname{ILSD}\left(\theta ; \beta_{0}, \beta_{1}, \beta_{2}\right)$ for some non-zero numbers $\theta, \beta_{0}, \beta_{1}$, and $\beta_{2}$, then its PGF, $G(t)$, is given by (51), from which we get that;

$$
\beta \frac{\log (1-\theta t)}{\log (1-\theta)}=G(t)-\beta_{0}-\beta_{1} t-\beta_{2} t^{2}
$$

Differentiating (51) with respect to t, we have that;

$$
\frac{\partial}{\partial \mathrm{t}} \mathrm{G}(\mathrm{t})=\beta_{1}+2 \beta_{2} \mathrm{t}-\frac{\theta \beta}{(1-\theta \mathrm{t}) \log (1-\theta)} .
$$

Hence, using (53) into (54), we get that $G(t)$ satisfies (52) with; $a=1, b=-\theta, c=\beta_{1}-\frac{\theta \beta}{\log (1-\theta)}, d=2 \beta_{2}-$ $\theta \beta_{1}$ and $e=2 \beta_{2} \theta$. If $\theta, \beta_{1}$ and $\beta_{2}$ are chosen such that $\frac{\theta}{\log (1-\theta)} \neq \frac{\beta_{1}}{\beta}$, then $\mathrm{c}$ is a non-zero number, and that $\theta \neq \frac{2 \beta_{2}}{\beta_{1}}$, then $d$ is also a non-zero number, and since $\mathrm{a}=1, \mathrm{~b}=-\theta$ and $\mathrm{e}=2 \beta_{2} \theta$, are all non-zero numbers. ii) Without loss of generality, let us assume that $a=1$, hence (52) can be written as, with $d$ replaced by $h$ in order not to be confused with derivative symbol d;

$$
\frac{\partial}{\partial t} G(t)=\frac{c+h t+e t^{2}}{1+b t}
$$

from which we have;

$$
G(t)=\int \frac{c+h t+e t^{2}}{1+b t} d t+k_{1}
$$

where $\mathrm{k}_{1}$ is an arbitrary constant. Since;

$$
\frac{c+h t+e t^{2}}{1+b t}=\frac{b^{2} c-b h+e}{b^{2}(1+b t)}+\frac{b h-e}{b^{2}}+\frac{e t}{2 b}
$$

it follows that the integral in (55) can be easily evaluated, from which, with $d$ replaced by $h$, we have that; 


$$
G(t)=\frac{\left(b^{2} c-b h+e\right)}{b^{3}+k_{2}} \log (1+b t)+\frac{(b h-e) t}{b^{2}}+\frac{e t^{2}}{2 b}
$$

where $k_{2}$ is an arbitrary constant. Since $G(1)=1$; then $\mathrm{k}_{2}$ can be found, and then, we can write that $\mathrm{G}(\mathrm{t})$ in a form as given by (51) with;

$$
\begin{gathered}
\theta=-b \\
\beta_{0}=1-\left(\frac{d}{b}-\frac{e}{b^{2}}\right)-\frac{e}{b}-\left(\frac{e}{b^{3}}-\frac{d}{b^{2}}+\frac{c}{b}\right) \log (1+b) \\
\beta_{1}=\frac{d}{b}-\frac{e}{b^{2}} \\
\beta_{2}=\frac{e}{2 b}
\end{gathered}
$$

and

$$
\beta=1-\beta_{0}-\beta_{1}-\beta_{2}
$$

Let us consider possible values of the parameters $\theta, \beta_{0}$, $\beta_{1}, \beta_{2}$ and $\beta$ given by (56) to (60), respectively.

Now if; $-1<\mathrm{b}<0$, then $\theta$ satisfies that; $0<\theta<1$. If $-2 \mathrm{~b}<\mathrm{e}<0$, then $0<\frac{\mathrm{e}}{2 \mathrm{~b}}<1$, hence $\beta_{2}$ satisfies that $0<\beta_{2}<1$. If $\mathrm{b}+\frac{\mathrm{e}}{\mathrm{b}}<\mathrm{d}<\frac{\mathrm{e}}{\mathrm{b}}$, then $0<\frac{\mathrm{d}}{\mathrm{b}}-\frac{\mathrm{e}}{\mathrm{b}^{2}}<1$, hence $\beta_{1}$ satisfies that $0<\beta_{1}<1$.

Consider now $\beta$ as given by (60), and note that $\beta=\left(\frac{e}{b^{3}}-\frac{d}{b^{2}}+\frac{c}{b}\right) \log (1+b)$, and since if c satisfies that $-\left(\frac{e}{b^{2}}-\frac{d}{b}\right)<c<\frac{b}{\log (1+b)}-\left(\frac{e}{b^{2}}-\frac{d}{b}\right)$, then $0<\beta<1$, and so also $0<1-\beta<1$, or equivalently; $0<\beta_{0}+$ $\beta_{1}+\beta_{2}<1$, from which we arrive to that $0<\beta_{0}<1$, since $0<\beta_{1}<1$ and $0<\beta_{2}<1$. This completes the proof of the Theorem.

Note that, c cannot assumed to be zero in (52), since if it does, then the solution of (52) will be exactly the same form as given in the solution of (52), with $c=0$ in (57). The values of the parameters $b$ and e can be found but there is no real number $d$ that can satisfies both $0<\beta_{0}<$ 1 and $0<\beta_{0}+\beta_{1}+\beta_{2}<1$.

Theorem 10 leads to the followings.

Theorem 11 (Alshkaki (2016d), p 3): Let X be a discrete rv taking non-negative integer values, then $X \sim$ $\operatorname{ZOILSD}\left(\theta ; \beta_{0}, \beta_{1}\right)$, for some non-zero numbers $\theta, \beta_{0}$ and $\beta_{1}$ if and only if its PGF, G(t), satisfies for some non-zero numbers $a, b, c$ and $d$.

$$
(a+b t) \frac{\partial}{\partial t} G(t)=c+d t
$$

Proof: Just let $\mathrm{e} \rightarrow 0$ in Theorem 10.

Theorem 12 (Alshkaki (2016e), p 3): Let X be a discrete rv taking non-negative integer values, then $X \sim$ $\operatorname{ZOILSD}\left(\theta ; \beta_{0}\right)$, for some non-zero numbers $\theta$ and $\beta_{0}$ if and only if its PGF, $G(t)$, satisfies for some non-zero numbers $\mathrm{a}, \mathrm{b}$ and $\mathrm{c}$ that;

$$
(\mathrm{a}+\mathrm{bt}) \frac{\partial}{\partial \mathrm{t}} \mathrm{G}(\mathrm{t})=\mathrm{c}
$$

Proof: Just $\mathrm{d} \rightarrow 0$ and $\mathrm{e} \rightarrow 0$ in Theorem 10 .

\section{Conclusions}

The Poisson, the binomial, the negative binomial, and the logarithmic series distributions, are well known discrete distributions members of the class of power series distributions. We have introduced a sub-class of the class of power series distributions, namely, a finite k-points inflated distributions of this class. Mathematical properties, such as, the mean, variance, and probability generating function of the general k-points inflated power series distribution in terms of their general standard non-inflated distribution has been considered. Finally, for each of these well-known discrete distributions; the Poisson, the binomial, the negative binomial, and the logarithmic series distributions, we give a characterization of their first 3-points (zero, one and two) inflated distributions through a given linear differential equation of their probability generating function. These characterizations result also give the characterization of their zero inflated and zero-one inflated distributions as well.

We would strongly recommend researcher to use this result to other distributions, as well as, to consider some other forms.

\section{REFERENCES}

[1] Abdul-Razak, R. S. and Patil, G. P. (1986). Power series distributions and their conjugates in stochastic modeling and Bayesian inference. Communication in Statistics, Part 1 - Theory and Methods, 15(3), 623-641.

[2] Abdul-Razak, R. S. and Patil, G. P. (1994). Some stochastic characteristics of the power series distributions. Pakistan Journal of Statistics, 10(1), 189-203.

[3] Alshkaki, R. S (2016a). Characterization of the Zero-One Inflated Poisson Distribution, Sch. J. Phys. Math. Stat. 2016; 3(4):123-126. DOI: 10.21276/sjpms.2016.3.4.1.

[4] Alshkaki, R. S. (2016b). A Characterization of the Zero-One Inflated Binomial Distribution, Journal of Research in Applied Mathematics, Volume 2, Issue 12, 4-07.

[5] Alshkaki, R. S. (2016c). A Characterization of the Zero-One Inflated Negative Binomial Distribution, Research Journal of Mathematical and Statistical Sciences, Vol. 4(9), 1-3.

[6] Alshkaki, R. S. (2016d). A Characterization of the Zero-One Inflated Logarithmic Series Distribution, International Journal of Scientific Research in Mathematical and Statistical Sciences, Volume-3, Issue-4, 1-3.

[7] Alshkaki, R. S. (2016e). A Characterization of the Zero-Inflated Logarithmic Series Distribution. International Journal of Mathematics and Statistics Invention, Volume 4 Issue 7, 1-3.

[8] Edwin, T. K. (2014). Power series distributions and zero-inflated models. Ph.D. Thesis, University of Nairobi. 
[9] Hu, Mei-Chen, Pavlicova, M. and Nunes, E. V. (2011). Zero-Inflated and Hurdle Models of Count Data with Extra Zeros: Examples from an HIV-Risk Reduction Intervention Trial, The American Journal of Drug and Alcohol Abuse, 37:5, 367-375.

[10] Johnson, N. L., Kemp, A. W. and Kotz, S. (2005). Univariate Discrete Distributions, Third Edition, John Wiley and Sons; New Jersey.

[11] Leroux, B. G., and Puterman, M. L. (1992). Maximum-Penalized-Likelihood Estimation for Independent and Markov-Dependent Mixture Models. Biometrics, 545-558.

[12] Lukusa, T. M., Lee, S. and Li C. (2017). Review of Zero-Inflated Models with Missing Data, Current Research in Biostatistics 2017, 7 (1): 1-12.

[13] Nanjundan, G. and Pasha, S. (2015a). A Note on the Characterization On the Zero-Inflated Poisson Distribution. Open Journal of Statistics, 5, 140-142.

[14] Nanjundan, G. and Pasha, S. (2015b). A Characterization of Zero-Inflated Binomial Distribution. International Journal of Mathematics and Computer Research, Vol. 3, No. 11, 1187-1190.

[15] Phang, Y. N. and Loh, E. F. (2013). Zero Inflated Models for Overdispersed Count Data, International Journal of Health and Medical Engineering, Vol:7, No:8, 1331-1333.

[16] Sellers, K. F. and Raim, A. (2017). A flexible zero-inflated model to address data dispersion, Computational Statistics and Data Analysis, 99 (2016) 68-80.

[17] Suresh, R., Nanjundan, G., Nagesh, S., and Pasha, S. (2015). On a Characterization of Zero-Inflated Negative Binomial Distribution. Open Journal of Statistics, 5, 511-513. http://dx.doi.org/10.4236/ojs.2015.56053.

[18] Yang, S., Harlow, L. L., Puggioni, G. and. Redding, C. A. (2017). A Comparison of Different Methods of Zero-Inflated Data Analysis and an Application in Health Surveys, Journal of Modern Applied Statistical Methods, May 2017, Vol. 16, No. 1, 518-543.

[19] Zamri, N. S. N. and Zamzuri, Z. H. (2017). A Review On Models For Count Data With Extra Zeros, AIP Conference Proceedings 1830, $080010 \quad$ (2017); https://doi.org/10.1063/1.4980994 